\title{
A matter of time
}

\section{Although still in its infancy, attosecond science has already captured the imagination of the scientific community with its promise of enabling rapidly evolving phenomena in nature to be investigated.}

Since the first demonstration of isolated attosecond $\left(10^{-18} \mathrm{~s}\right)$ pulses in 2001, the field of attosecond science has progressed rapidly, enhancing our understanding of ultrafast electronic processes in atoms, molecules and condensed matter. Attosecond photonics - currently one of the most promising branches of modern photonics - is undoubtedly progressing at an extremely fast pace.

This issue features a focus on attosecond photonics with four Reviews and a Commentary providing an overview of all aspects of this young but rapidly progressing field.

A broad spectral bandwidth and control of the spectral phase are essential for generating extremely short isolated attosecond pulses. In 2012, light pulses as short as 67 as were generated and broadband attosecond continua covering extreme ultraviolet and soft $\mathrm{X}$-ray wavelengths were also achieved. On page 178, Chini, Zhao and Chang review significant recent advances in the generation, characterization and applications of ultrabroadband, isolated attosecond pulses whose spectral bandwidths are comparable with their central frequency. They envisage that combining the techniques used to generate broadband attosecond pulses with those for generating high-energy isolated attosecond pulses will allow single-cycle pulses to be generated that have gigawatt peak powers and petawatt per square centimetre peak intensities at the target; such pulses will be useful for true attosecond-pumpattosecond-probe experiments.

However, because attosecond pulses in the vacuum ultraviolet, extreme ultraviolet and soft X-ray regions are currently the shortest of all probe pulses, it is challenging to measure them. On page 187, Kim, Villeneuve and Corkum compare and contrast attosecond measurements based on photorecombination (in situ methods) and photoionization (ex situ methods). The former process involves gently perturbing the electron responsible for attosecond pulse formation, whereas in the latter process attosecond pulses produce photoelectrons by photoionization in a second medium and a synchronized laser field perturbs the photoelectron spectrum.

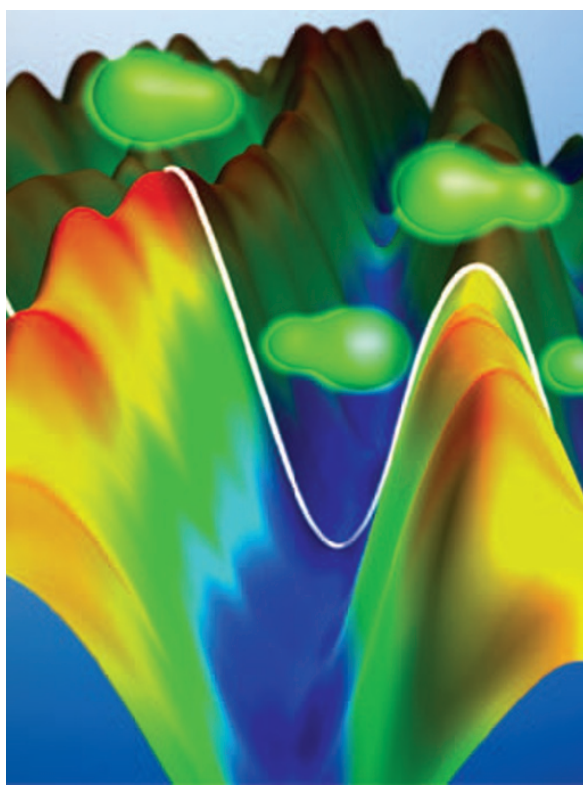

for the future evolution of information technology. These applications stand out from others in that they have the potential to produce great discoveries of both fundamental importance and great technological relevance. On page 205, Krausz and Stockman detail the basic concepts underlying attosecond measurement and control techniques. Their Review emphasizes the exploration of the fundamental speed limit of electronic signal processing, and addresses how controlled light fields and attosecond measurement techniques may be instrumental in pushing the speed frontier of digital electronics.

By a fortuitous coincidence, page 214 of this issue features a Letter by Tim Paasch-Colberg and colleagues on a solidstate light-phase detector that can detect the absolute offset between the carrier wave and envelope (the carrier-envelope phase) of an ultrashort pulse. The device is promising for routine measurement and monitoring of the carrier-envelope phase in attosecond experimental set-ups, making it a useful addition to the toolbox of attosecond metrology and spectroscopy.

The community is currently discussing the question of whether attosecond science really does measure processes in real time. The Commentary by Leone and coauthors on page 162 points out that a deep understanding of attosecond time processes is inextricably linked to distributions in times of arrival, detailed phase-shift information and interferences between channels. The lasting impact will be an understanding of how short-time dynamics can determine the electronic properties of more complex systems.

In the first decade of attosecond science, experiments have been constrained by the low intensity of available attosecond pulses. However, rapid progress has been made in the production of higher-flux lasers with ultrabroadband spectra and better highharmonic conversion schemes, as well as free-electron lasers. It is just a matter a time before we witness how these new-gained capabilities can be harnessed to track and control electrons in atoms, molecules and condensed matter enabling problems of direct relevance to life, technology and, potentially, medicine to be investigated. 